\title{
Pasantías PENTA UC: Una Propuesta Innovadora de Desarrollo Profesional Docente
}

\section{PENTA UC Internships: An Innovative Proposal for Teacher Training}

\author{
Catalina García y Violeta Arancibia \\ Pontificia Universidad Católica de Chile
}

\begin{abstract}
Se evaluaron procesos y resultados obtenidos durante la implementación de un nuevo modelo de perfeccionamiento docente, Pasantía PENTA UC, que propone que profesores asistan como alumnos, durante un semestre, a un curso dictado por académicos universitarios para escolares con talentos académicos, y así profundicen en contenidos de sus disciplinas y en herramientas metodológicas, y elaboren un proyecto de transferencia de lo aprendido al aula regular. Los resultados muestran que los profesores aumentan sus conocimientos, aprenden herramientas metodológicas, reflexionan sobre el proceso de aprendizaje y desarrollan proyectos de calidad y viables. Así, la Pasantía PENTA UC se vislumbra como una opción potencialmente efectiva de perfeccionamiento.
\end{abstract}

Palabras Claves: perfeccionamiento docente, evaluación, pasantía PENTA UC.

\begin{abstract}
Procedures and outcomes obtained during the implementation of a new teacher professional development model, Pasantía PENTA UC, were evaluated, this internship proposes that teachers attend, as students, during a semester, to a course dictated by university professors to gifted students, and thus getting in-depth knowledge in their areas of expertise and acquiring new methodological tools, and to create a transference project from the internship to the regular classroom. The results show that the teachers increase their knowledge, learn methodological tools, reflect over the learning process, and develop quality and viable projects. Thus, Pasantía PENTA UC outstands as a potentially effective improvement option.
\end{abstract}

Keywords: teacher training, evaluation, PENTA UC internships.

Distintos estudios plantean que los profesores tienen un importante impacto en el aprendizaje escolar (Brunner \& Elaqua, 2003; Scheerens, 2000), y por ello existe gran interés en su tarea profesional y en su perfeccionamiento (Bellei, 2001; Bellei \& Pérez, 2000; Fullan \& Stiegelbauer, 1997; OCDE, 2004; Stiegler \& Hierbert, 2002).

Catalina García, Centro de Estudios y Desarrollo de Talentos, Pontificia Universidad Católica de Chile.

Violeta Arancibia, Centro de Estudios y Desarrollo de Talentos, Pontificia Universidad Católica de Chile.

La correspondencia relativa a este artículo deberá ser dirigida a las autoras, Centro de Estudios y Desarrollo de Talentos, Pontificia Universidad Católica de Chile, Av. Vicuña Mackenna 4860, Macul, Santiago, Chile. E-mail: cigarcia@uc.cl,varancib@uc.cl

Esta investigación fue realizada en el marco del proyecto FONDEF DO2I1039, Evaluación de un Modelo de Identificación y Educación de Escolares con Talentos Académicos, para su transferencia a organismos educacionales en Chile y Latinoamérica, llevado a cabo entre los años 2003 y 2005, y constituyó la tesis para optar al grado de Magíster en Psicología, mención Psicología Educacional de la Pontificia Universidad Católica de Chile. Esta tesis fue realizada con el apoyo de CONICYT.
Sin embargo, las ofertas de perfeccionamiento no se han acompañado de mejorías en el aprendizaje de los estudiantes (Arancibia, 1994; Bellei, 2001; Fullan \& Stiegelbauer, 1997). "La opinión más extendida es que el impacto de los programas para incrementar la competencia de los profesores, y cambiar la enseñanza es bastante limitado" (Marchesi \& Martin, 1998, p. 154).

Pero si bien la investigación muestra consistentemente la inefectividad de los perfeccionamientos (Arancibia, 1994; Ávalos, 2004; Guskey, 2002), no habrá mejoras educativas sin un desarrollo profesional continuo. No se trata de no hacer perfeccionamiento, sino de hacerlo diferente. Según Ávalos (2004), Chile ha buscado mejorar el perfeccionamiento pero los resultados no han sido satisfactorios.

El Centro de Estudios y Desarrollo de Talentos de la Universidad Católica, creó las Pasantías PENTA $\mathrm{UC}$, que en el contexto de la educación de talentos académicos, buscan aportar a través de un modelo de perfeccionamiento que contribuya al aprendizaje de contenidos disciplinarios y de herramientas metodológicas, y promueva la transferencia de estos aprendizajes a su práctica pedagógica regular. 
El presente artículo describe el modelo de $\mathrm{Pa}-$ santía PENTA UC. Luego, se dan a conocer los resultados obtenidos de su evaluación. Finalmente, se plantean conclusiones y comentarios a partir de estos resultados.

\section{Antecedentes}

\section{Pasantias PENTA UC}

El Centro de Estudios y Desarrollo de Talentos de la Pontificia Universidad Católica de Chile, desarrolla el Programa Educacional para Niños con Talentos Académicos PENTA UC, para alumnos entre $6^{\circ}$ básico y IV medio, que destaquen por su potencial y habilidades intelectuales, ofreciéndoles un programa de enriquecimiento extracurricular. Los alumnos participan de cursos a su elección dictados por académicos universitarios, en diferentes áreas (biología, física, química, historia, lenguaje, matemáticas, ciencias sociales y otras), utilizando las instalaciones de la Universidad. Los cursos son dictados los viernes de 15:00 a 18:00 hrs. y los sábados de 9:00 a 12:00 hrs. Este programa ha constatado la experticia y efectividad de sus profesores mostrando resultados positivos en el aprendizaje de los alumnos ${ }^{1}$.

A partir de esta experiencia, se diseñaron las Pasantías PENTA UC, que buscan contribuir al perfeccionamiento y actualización disciplinaria y metodológica de los docentes, así como transferir innovaciones en diseño curricular, metodologías de enseñanza y prácticas pedagógicas.

\section{Objetivos de la Pasantía PENTA UC}

1. Contribuir al perfeccionamiento y actualización disciplinaria de profesores.

2. Dar a conocer herramientas y estrategias metodológicas que permitan enriquecer la labor docente.

3. Promover la transferencia de los contenidos y estrategias desarrolladas por el PENTA UC, a las clases regulares de los profesores.

\section{Metodología}

Concurso. Se abre una oferta para profesores, quienes deben presentar una ficha personal indicando el curso de interés, un currículum vitae, un ensayo

1 Mayor información del programa PENTA UC en www.pentauc.cl con sus motivaciones para participar y una carta del Director/a autorizando su participación y comprometiéndose a apoyar la implementación del proyecto.

Selección de pasantes. Se aplica una rúbrica para seleccionar a los profesores más motivados, siendo considerado que los temas ofrecidos sean coherentes con los intereses de los profesores. Los profesores seleccionados participan de un programa de 96 horas cronológicas.

Pasantes eligen un curso PENTA UC. Diseñado para alumnos con talento académico, que trata un tema en profundidad dentro de su disciplina, y asisten a 15 sesiones de clase de 3 horas, como observadores-participantes, siendo compañeros de alrededor de 25 escolares talentosos, y accediendo a bibliografía. Cada curso PENTA UC tiene un máximo de 2 pasantes.

Asistencia a 4 asesorías disciplinarias. Con el académico del curso PENTAUC, para discutir y aclarar contenidos del curso con expertos, revisar la bibliografía entregada, reflexionar sobre los procesos de enseñanza-aprendizaje observados y experimentados, y revisar el proyecto.

Asistencia a 3 asesorías metodológicas grupales y 2 individuales. Con expertos en psicoeducación, para recibir orientación y retroalimentación sobre su proyecto de transferencia, así como orientación y guía en la experiencia como alumno observador participante.

Diseño y elaboración del proyecto de transferencia. Cada profesor va desarrollando su propuesta para transferir lo aprendido a su clase regular.

Este modelo consideró una revisión de aspectos favorables y desfavorables para los perfeccionamientos planteados en la literatura, siendo los antecedentes que subyacen a la Pasantía PENTA UC los que se mencionan a continuación: (Arancibia \& García, 2003).

\section{Fundamentación Teórica}

Según Núñez (2000), los perfeccionamientos no cuentan con modelos teóricos explícitos y evaluados. Falta conocimiento específico sobre los profesores y su práctica, y los estándares que deben alcanzar (Alliaud \& Duschatzky, 1998; Sternberg \& Horvarth, 1995 citados en Jones, 1997). La Pasantía cuenta con un marco teórico que permite una planificación y aplicación que favorece su efectividad (Fullan \& Stiegelbauer, 1997; Núñez, 2000). Entre los elementos fundamentales, se considera lo siguiente:

Aprendizaje en adultos. Según Undurraga (2004), se caracteriza por la diversidad de experiencias que 
influirán en los aprendizajes, y por la posibilidad de negociar estas experiencias de aprendizaje. La Pasantía considera metas realistas y relevantes para los profesores, quienes conocen los objetivos y se plantean metas personales. Se trabaja en pequeños grupos y en instancias en que los profesores reciben feedback, elementos recomendados para el éxito del perfeccionamiento (Project ExCELL, 1997).

Cambio docente. La Pasantía considera asesorías que acompañan el proceso, ayudando a descubrir alternativas de acción exitosas y acogiendo temores, pues el trabajo extra que demandan los cambios genera ansiedad (Guskey, 2002) y la sensación de soledad de los profesores, el sentirse limitados por la estructura escolar y la falta de tiempo, obstaculiza los cambios (Cashion \& Sullegner, 2000). Según Ferrández, Gairín y Tejada (1990) es necesario permitirles aprender a través de la participación, para disminuir resistencias dado su temor al fracaso, a mostrar desconocimiento y a la incertidumbre.

Tiempo para aprender. Esta Pasantía tiene una duración de 4 meses con el objetivo de permitir un tiempo apropiado para planear y aprender nuevas prácticas (Pink, 1989 citado en Fullan \& Stiegelbauer, 1997). Además, se realiza en paralelo con la práctica docente, considerando que para introducir cambios en la práctica se necesita mantener el compromiso con el cambio y tener evidencia de éxito (Bandura, 1983; Cashion \& Sullegner, 2000; Van Der Sijde, 1989). Los pasantes pueden poner a prueba elementos aprendidos en la Pasantía, en su clase cotidiana. La Pasantía PENTA UC se plantea en un semestre, con asesorías y reflexión permanente que acompañan el proceso de aprendizaje, abriendo espacios para sus inquietudes y para recibir retroalimentación.

Aprendizaje por observación y en la práctica. El profesor observa a un académico universitario experto, haciendo clases a alumnos escolares, y observa a los alumnos responder a ello, presentando un modelo válido (Bandura, 1983). Los profesores participan de estas actividades como un alumno más, pues según Levine (2002), no se puede aprender si no hay práctica. Ambos aspectos son cruciales, pues los perfeccionamientos exitosos acompañan la teoría con demostración, práctica, retroalimentación y entrenamiento (Jungsatikul, 1991 citado en Arancibia, 1994). Esta experiencia permite al pasante ponerse en el lugar de los estudiantes y compartir con ellos la experiencia de aprender, fundamental para que el análisis y la búsqueda de los cambios sea atingente, entendiendo que enseñanza y aprendizaje son parte del mismo fenómeno.

\section{Necesidades y Contexto Particular de los} Profesores

Usualmente los perfeccionamientos son dirigidos a individuos desligados de su contexto laboral (Arancibia, Herrera \& Strasser, 1999), y los temas no son seleccionados por los profesores (Guskey, 2002; Núñez, 2000). Considerar las necesidades de los profesores impactará en su motivación haciendo los perfeccionamientos más efectivos (Project ExCELL, 1997). Es por esto que la Pasantía PENTA UC incorpora lo siguiente:

Compromiso institucional. Se solicita una carta del Director para apoyar la participación del profesor y la implementación de un proyecto en el establecimiento, pues el apoyo de la institución escolar es necesario para las innovaciones (Barocio, 1993).

Objetivos claros y conocidos por los profesores. Los profesores conocen los objetivos y procedimientos, generando un compromiso informado, pues es crucial para que puedan focalizar sus esfuerzos y recursos cognitivos para lograr los resultados esperados (Bandura, 1983; Van Der Sijde, 1989).

Aprendizaje significativo. Según Rodgers (2002), el inicio más productivo para el desarrollo docente es su propia experiencia. Se da la posibilidad de crear un proyecto basado en la propia experiencia y motivación, aumentando las posibilidades de modificar la práctica (Arancibia, 1994; Marchesi \& Martin, 1998).

\section{Estrategias de Aprendizaje Apropiadas}

Fullan (1991 citado en Marchesi \& Martin, 1998) señala que habitualmente los seminarios son numerosos, intensivos, rápidos y breves. Según Gimeno (1998 citado en Alliaud \& Duschatzky, 1998), es necesario un cambio de metodología incorporando la inserción en la práctica y el perfeccionamiento en ejercicio, que hacen posible conectar la teoría con la práctica, y favorecen la reflexión en la acción. Los perfeccionamientos utilizan metodologías expositivas (Arancibia, Herrera \& Strasser, 1999), que no logran transformar las prácticas. Atendiendo a ello, esta Pasantía incorpora lo siguiente:

Asesorías e interacción con pares. Espacios de trabajo individual y grupal, con docentes expertos y con sus pares, para dar sugerencias, compartir conocimientos y discutir ideas, aumentando las posibilidades de modificar la práctica (Arancibia, 1994; Lieberman \& Miller, 1999; Marchesi \& Martin, 1998). Cuentan con la oportunidad de presentar y discutir 
sus aprendizajes y proyectos con otros pasantes, quienes pueden aportar elementos de reflexión y nuevas ideas. Además, participan de asesorías individuales con asesores especializados y con el docente experto de su tema particular, realizando en estas instancias análisis guiado de lo aprendido y selección de metas de mejoramiento a través de los proyectos; recibiendo retroalimentación y orientación sobre los avances y aspectos por mejorar, lo que es favorable para el aprendizaje (Kerwin \& Rhoads, 1996). Contar con retroalimentación sobre el progreso y las acciones exitosas es reforzante, y se necesita para tolerar la ansiedad y la frustración de los errores al iniciar los cambios (Guskey, 2002).

Reflexión docente. El éxito depende en gran medida del grado en que los profesores reflexionan sobre sus problemas y quieren resolverlos (Stallings, 1989 citado en Fullan \& Stiegelbauer, 1997). La Pasantía PENTA UC incorpora asesorías que orienten y fomenten la reflexión sobre lo aprendido, así como guías de trabajo personales de reflexión sobre maneras de mejorar la práctica. Se apunta a una reflexión guiada y continuada en torno a elementos significativos (Barocio, 1993). Es fundamental que la observación y el hacer estén de la mano de pensar sobre dichas experiencias pues solo de ese modo es posible aprender realmente de ellas (Levine, 2002; Marchesi \& Martin, 1998; Project ExCELL, 1997; Stallings, 1989 citado en Fullan \& Stiegelbauer, 1997).

Apoyo y seguimiento. La Pasantía incorpora una evaluación de seguimiento a los profesores pasantes, pues pocas veces existe seguimiento y apoyo a las ideas y prácticas posteriores a los programas, lo que es necesario para fortalecer los cambios (Cashion \& Sullegner, 2000; Fullan, 1991 citado en Marchesi \& Martin, 1998; Pink, 1989 citado en Fullan \& Stiegelbauer, 1997).

\section{Objetivos de aprendizaje adecuados}

El perfeccionamiento debe asegurar que los profesores adquieran conocimientos y técnicas para tomar decisiones educativas efectivas. Dados los avances tecnológicos, se demandan nuevas habilidades, jugando un rol central la capacitación continua (Brunner \& Elaqua, 2003). Rodgers (2002), plantea que los profesores deben cambiar su mirada desde la enseñanza, al aprendizaje de los estudiantes. Ello requiere de profesionales expertos.

Profesores como profesionales. Contar con profesionales de alta calidad es central para el mejoramiento educativo (Guskey, 2002). La profesiona- lización implica estar en constante perfeccionamiento (Núñez, 2004) y contar con habilidad y autonomía para juzgar cuándo y cómo utilizar los conocimientos (Arancibia, 1994; Darling-Hammond et al., 1983 citado en Marchesi \& Martin, 1998). Las políticas educativas chilenas a partir de los 90 han apuntado a la profesionalización docente a través de la formación en servicio, sin resultados positivos (Ávalos, 2004; Bellei \& Pérez, 2000; MINEDUC, 2001; Núñez, 2004; OCDE, 2004). Lo que se requiere es replantear la formación inicial y en servicio, con propuestas profundas y de largo plazo (OCDE, 2004).

La Pasantía procura que los profesores reconozcan la necesidad de perfeccionarse y aprender permanentemente, para tomar decisiones en base a su conocimiento teórico y de la situación educativa particular a la que se enfrentan. Estas habilidades son potenciadas a través de las asesorías y la elaboración del proyecto de transferencia.

Ser profesional implica ser evaluado y dar cuenta de lo que se hace, por ello la Pasantía somete a los profesores a la experiencia permanente de evaluar y ser evaluado, por sus pares, por los docentes PENTA $\mathrm{UC}$, por los asesores, y por ellos mismos. Deben poder fundamentar las decisiones educativas y discutirlas con otros.

Profesores como expertos. La Pasantía busca realzar aquellos conocimientos particulares que distinguen a los profesores y por ello, considera el conocimiento metodológico tanto como de contenido. El primero suele ser considerado el conocimiento de contenidos a enseñar y es central pues distingue a profesores expertos y efectivos (Arancibia, Herrera \& Strasser, 1999; Jones, 1997).

La Pasantía permite participar de un curso que trata una temática específica dentro de su ámbito de enseñanza, de manera que pueda profundizar en dichos contenidos. A partir de ello el profesor debe diseñar un proyecto de transferencia bajo la premisa de que sólo se puede ser un buen profesor si se sabe muy bien lo que debe enseñarse. El pasante debe participar de las clases, realizar cada una de las actividades y revisar textos de profundización.

Junto con ello, deben observar, comentar y analizar las prácticas pedagógicas utilizadas en la enseñanza de dichos contenidos, de modo de hacerse cada vez más expertos en la elección de metodologías adecuadas a los aprendizajes esperados y a las características de los estudiantes.

Ir en la línea de la experticia es necesario para mejorar la calidad de las prácticas docentes, pues los expertos notan aspectos de la clase que no no- 
tan los novatos y son más selectivos en el uso de la información, han adquirido un conocimiento extenso, y organizan, representan e interpretan la información en torno a conceptos clave, liberando recursos cognitivos para responder a imprevistos. Su atención está en las respuestas de los alumnos y en lograr los objetivos de aprendizaje (Bransford, Brown \& Cocking, 2000; Livingston \& Borko, 1989).

En resumen, la Pasantía PENTA UC cuenta con características que favorecen el aprendizaje docente. Los pasantes escogen el curso que les motiva, se da la posibilidad de reflexión y aprendizaje (clase a clase, en asesorías, con el proyecto), observan a un experto haciendo clases, accediendo a estrategias de enseñanza-aprendizaje, y aprenden en la práctica, al tomar el rol de alumno. Se considera el contexto del profesor para transferir aprendizajes a su práctica regular, exigiéndose un proyecto de transferencia que defina estas acciones de transferencia, y se involucra el apoyo del Director/a de su establecimiento. Tiene una duración de un semestre, paralelo a sus clases cotidianas, y se realiza siendo "compañero" de escolares, tres características innovadoras.

Esta investigación evaluó si este modelo logra sus objetivos, y qué elementos del proceso son relevantes para lograr esos resultados, para tomar decisiones sobre nuevas implementaciones y analizar los impactos de esta experiencia, recopilados en una segunda investigación. Específicamente, se buscó conocer (a) si los profesores aprendieron contenidos en su disciplina particular de enseñanza; (b) si aprendieron herramientas metodológicas; (c) si el proyecto de transferencia se constituye en un método apropiado para promover la transferencia del aprendizaje a su labor docente; (d) la evaluación de los propios pasantes con respecto a la experiencia de Pasantía PENTA UC; y (e) alguna tendencia que mostrara relación entre las características de los pasantes y los resultados obtenidos.

\section{Método}

\section{Participantes}

Se trabajó con 20 profesores que cursaron una Pasantía PENTA UC durante el $2^{\circ}$ semestre del 2003. La muestra se compone de 9 hombres y 11 mujeres pertenecientes a 12 colegios.

\section{Instrumentos}

Para la recolección de datos se elaboraron diferentes instrumentos de evaluación. Estos fueron: (a) prueba de conocimiento disciplinario; (b) pauta de evaluación de asesorías metodológicas; (c) pauta de evaluación de asesorías disciplinarias; (d) pauta de evaluación de proceso de pasantes; (e) encuesta de satisfacción de pasantes; y (f) pauta de análisis de proyecto de transferencia. Se presenta una descripción de éstos en la Tabla 3.

\section{Procedimiento}

Este estudio se realizó entre agosto y diciembre del 2003. Se utilizó metodología cualitativa y cuantitativa descriptiva. Se realizaron reuniones con pasantes, docentes PENTA UC y psicóloga PENTA UC, para solicitar su colaboración y dar a conocer los instrumentos. Para la recolección de datos se realizó lo siguiente:

Aplicación de prueba de conocimientos a pasantes. En la primera y última sesión. Se obtuvieron porcentajes de logro, analizando las diferencias entre ambas evaluaciones.

Tabla 1

Muestra de pasantes según dependencia escolar

\begin{tabular}{ccccc}
\hline Dependencia & Municipal & Subvencionado & Particular & Total \\
\hline $\begin{array}{c}\text { Número } \\
\text { profesores }\end{array}$ & 13 & 1 & 6 & 20 \\
\hline
\end{tabular}

Tabla 2

Muestra de pasantes según área disciplinaria en que cursaron la Pasantía PENTA UC

\begin{tabular}{cccccc}
\hline $\begin{array}{c}\text { Área } \\
\text { disciplinaria }\end{array}$ & $\begin{array}{c}\text { Ciencias } \\
\text { Biológicas } \\
\text { (química, } \\
\text { biología) }\end{array}$ & $\begin{array}{c}\text { Ciencias } \\
\text { Exactas } \\
\text { (física, } \\
\text { matemática) }\end{array}$ & $\begin{array}{c}\text { Humanidades } \\
\text { (lenguaje, } \\
\text { historia) }\end{array}$ & $\begin{array}{c}\text { Ciencias } \\
\text { Sociales } \\
\text { (psicología) }\end{array}$ & Total \\
\hline $\begin{array}{c}\text { Número } \\
\text { profesores }\end{array}$ & 5 & 6 & 5 & 4 & 20 \\
\hline
\end{tabular}


Tabla 3

Instrumentos de recolección de datos

\begin{tabular}{|c|c|c|}
\hline Instrumento & Objetivo & Características del instrumento y de la aplicación \\
\hline $\begin{array}{l}\text { Prueba de } \\
\text { conocimiento } \\
\text { disciplinario }\end{array}$ & $\begin{array}{l}\text { Conocer nivel de conocimiento } \\
\text { inicial y final de los profesores } \\
\text { pasantes, sobre los contenidos del } \\
\text { curso de Pasantía. }\end{array}$ & $\begin{array}{l}\text { Prueba y pauta de corrección elaborada por el docente } \\
\text { del curso de pasantía, experto en la disciplina, acorde a } \\
\text { los objetivos de aprendizaje definidos para el curso. } \\
\text { Consta de preguntas abiertas y/o cerradas, con } \\
\text { formato y extensión variable según los contenidos } \\
\text { de cada curso de pasantía. } \\
\text { Se aplica el primer día de clases y se repite el último día } \\
\text { de clases. }\end{array}$ \\
\hline
\end{tabular}

Pauta de evalua- Contar con información respecto de Protocolo que consta de tres preguntas de respuesta ción de asesorías los temas tratados durante las ase- abierta relacionadas con los temas tratados en la asemetodológicas sorías metodológicas. soría, comentarios, sugerencias y aspectos destacables Conocer aspectos relevantes sobre de los avances en la elaboración del proyecto. La terel proceso de elaboración de cera pregunta permite registrar otros comentarios soproyecto. bre la asesoría o el aprendizaje de los pasantes. Es respondido mensualmente por el asesor metodológico, al término de cada asesoría.

Pauta de evaluación de asesorías disciplinarias
Contar con información respecto de Protocolo que consta de tres preguntas de respuesta los temas tratados durante las ase- abierta relacionadas con los temas tratados en la asesorías disciplinarias. soría, aprendizajes disciplinarios, y otros comentarios Conocer aspectos relevantes sobre sobre la asesoría o el aprendizaje de los pasantes. el proceso de aprendizaje de los pa- Es respondido mensualmente por el docente PENTA santes.
Pauta de evalua- Conocer los aprendizajes ción de proceso de pasantes metodológicos reconocidos por el pasante y otros aprendizajes generales que quisieran transferir a su práctica docente.
UC, al término de cada asesoría.

Conocer reflexiones de los pasantes a partir de la experiencia de pasantía.

Cuestionario que consta de cuatro preguntas abiertas que indagan sobre aprendizajes y reflexiones relacionadas con el proceso de pasantía.

Cada pasante responde este cuestionario al término de cada mes de pasantía.
Encuesta de satisfacción pasantes
Conocer el grado de satisfacción de Encuesta de once preguntas cerradas sobre su nivel de los pasantes. satisfacción con diferentes aspectos de la pasantía, con respuesta de escala likert. Se solicita explicar el nivel asignado. Consta además de dos preguntas de respuesta abierta señalando aspectos positivos y negativos. Por último, consta de una pregunta de respuesta cerrada señalando si recomendarían la pasantía a otros profesores. Cada pasante responde este cuestionario al término de la pasantía.

Pauta de análisis Analizar si los proyectos de transfe- Pauta que permite registrar los elementos señalados de proyecto de rencia incorporan elementos seña- por los pasantes como aprendizajes en la pauta de evatransferencia lados por los pasantes como aprendizajes a lo largo de la pasantía. por los pasantes como aprendizajes en la pauta de eva-
luación de proceso. Se registra luego aquellos aspectos que aparecen incorporados en el proyecto de transferencia de los profesores pasantes.

Esta pauta es completada por el investigador. 
Registro de evaluación de asesorías metodológicas y disciplinarias. Al final de cada asesoría. Se obtuvieron categorías descriptivas sobre los temas tratados y los comentarios respecto al proceso de aprendizaje de los pasantes.

Registro proceso de aprendizaje de pasantes. Aplicada al término de cada mes de pasantía. Se obtuvieron categorías descriptivas de los aprendizajes metodológicos de los pasantes, y de sus reflexiones sobre los procesos de enseñanza aprendizaje.

Aplicación encuesta de satisfacción a pasantes. Al término de la Pasantía PENTA UC se solicitó a los pasantes responder esta encuesta. Se obtuvieron promedios en cada aspecto evaluado y categorías descriptivas de la justificación.

Análisis de proyectos de transferencia. Se revisó el diseño de cada proyecto, registrando los elementos que habían sido señalados como aprendizajes en las evaluaciones de pasantes.

Finalmente, se incorporó información sobre sexo, dependencia escolar y área disciplinaria analizando si mostraban alguna posible relación con el aprendizaje de contenidos, de metodologías, nivel de calidad del proyecto o satisfacción.

\section{Resultados}

La Pasantía PENTA UC se desarrolló según lo planificado. El porcentaje promedio de asistencia a los cursos de los pasantes fue $90 \%$ (entre 80 y $100 \%$ ). Participaron de 5 asesorías metodológicas y 4 disciplinarias, $y$ todos realizaron el proyecto. A continuación se presentan los resultados obtenidos en términos de los objetivos previamente señalados, integrando la información obtenida a partir de los diferentes instrumentos utilizados durante el estudio.

\section{Aprendizaje de Contenidos Disciplinarios}

Tabla 4

Porcentaje de logro de pasantes en prueba de conocimientos

\begin{tabular}{lcc}
\hline & \% logro inicial & \% logro final \\
\hline Promedio & 57.3 & 75.4 \\
DS & 17.4 & 18.1 \\
Mínimo & 31.8 & 34.1 \\
Máximo & 90.6 & 100.0 \\
\hline
\end{tabular}

Como se observa en la Tabla 4, los pasantes mejoraron significativamente su nivel de conocimiento en $18.2 \%$ de logro $(F=12.598 ; p<.002)$. Hubo 17 profesores $(85 \%)$ que mostraron mejorías, observándose gran heterogeneidad $(2.1 \%$ a $60 \%)$. Tres profesores no mostraron avances. Cabe señalar que la prueba utilizada fue elaborada por cada académi- co experto del PENTA UC, y no cuenta con un estudio de sus propiedades métricas.

Los pasantes señalaron que pudieron profundizar contenidos que no revisan con sus estudiantes, recordar conocimientos olvidados y relacionarlos con otros conocimientos. Se sorprendieron al reconocer cómo muchos de los contenidos están presentes en la vida cotidiana, lo que lo hace más significativo para los alumnos. La lectura de textos aportó a su mayor profundización y comprensión de los temas.

\section{Aprendizaje de Herramientas Metodológicas}

El 95\% de pasantes señala haber aprendido elementos metodológicos a partir de la experiencia de vivenciar la posición de alumno, y observar a otro docente. Estos aprendizajes estuvieron relacionados con cuatro ámbitos:

Proceso de enseñanza-aprendizaje. Destacan reconocer la importancia de "planificar las clases con objetivos claros, y dominar los temas a tratar". Agregan que el profesor debe "tener una actitud constante de evaluación y observación de los aprendizajes de los alumnos". Aprendieron que las actividades deben "permitir el aprendizaje activo de los estudiantes, permitirles preguntarse, experimentar y observar, y después entregarles contenidos y respuestas".

Manejo instruccional. Observan que es útil usar recursos que den independencia y libertad a los alumnos, promoviendo el trabajo grupal. Agregan que "el profesor debe tener un rol activo, explicitar claramente los objetivos y contenidos de la clase, profundizar y explicar lo que los mismos alumnos se preguntan" a partir de las actividades prácticas. Han observado que los docentes PENTA UC utilizan estrategias para motivar a los alumnos con diferentes recursos, rescatan las ideas de los estudiantes, y les dan autonomía en el trabajo, guiándolos al principio. Es decir, reconocen el rol activo del profesor al promover experiencias enriquecedoras para el aprendizaje de los estudiantes. Reconocen que esto tiene que ver con los contenidos tratados y las metodologías, así como con elementos del clima y factores emocionales de los estudiantes, como su atención y motivación. Pueden percibir el proceso de enseñanza aprendizaje como un fenómeno complejo del cual ellos, como profesionales, se hacen cargo. 
Relación con los alumnos. Aprendieron que "debe haber un clima en que a los alumnos se les respete y valore, dándoles más responsabilidades y libertad" para expresar lo que saben y quieren, reforzando lo positivo y considerando sus diferencias de opinión y de formas de aprender. Han aprendido que esto requiere de "reglas claras y guía por parte del profesor". Plantean que "es importante confiar en los alumnos", en sus capacidades y decisiones. Ser compañeros de los alumnos es crucial para mirarlos como personas con potencial, con ganas de aprender, con diferencias que llevan a desafiar al docente permanentemente.

Recursos pedagógicos. Observaron que existe una gran diversidad de recursos pedagógicos a su alcance que ellos no utilizan y que ayudan al aprendizaje, como ejemplos reales, lluvia de ideas, debates o mapas conceptuales, otros como obras de arte, música, y charlas de expertos. Destacan especialmente los recursos tecnológicos a los que pueden acceder, como computador, softwares, Internet y videos. Es decir, logran reconocer que la diversidad de recursos tiene que ver con su propia creatividad, y con un alto conocimiento de lo que se espera que los alumnos aprendan.

Un grupo de pasantes, señala cambios en ellos que les plantea enfrentar los procesos educativos de una nueva manera. Indican haberse "reencantado con la labor docente, lo que los motiva a hacer cosas nuevas". Tienen "nuevas expectativas y objetivos de aprendizaje, que lleven a sus alumnos a ser más autónomos en la búsqueda de conocimiento".

\section{Experiencia de Elaborar un Proyecto de Transfe- rencia como Herramienta para la Transferencia del Aprendizaje a la Labor Pedagógica Regular}

Cada pasante elaboró un proyecto para ser aplicado con alumnos de su establecimiento en el semestre a continuación, que fue evaluado por el docente PENTA UC, el asesor metodológico y el propio pasante, con una rúbrica diseñada para ello (máximo 18 puntos, aprobación 10). El puntaje final se obtuvo promediando las tres evaluaciones, el que se presenta en la Tabla 5, transformado a porcentaje de logro (donde 18 ptos. equivalen al 100\% de logro). Esta evaluación considera aspectos metodológicos así como de contenido.

Todos los pasantes aprobaron la Pasantía PENTA UC y desarrollaron un proyecto que cumple con los criterios de calidad y viabilidad requeridos, aunque se observa heterogeneidad en sus productos finales.

A través de los protocolos de registro, se aprecia que asesores metodológicos y disciplinarios destacan que los pasantes mostraron interés por aprender y compartir experiencias. Señalaron haber observado gran dificultad para estructurar un proyecto, especialmente para elaborar objetivos, actividades para lograrlos y métodos para evaluar el aprendizaje.

En promedio incorporaron a sus proyectos el $60 \%$ de los aprendizajes metodológicos, los que se señalan en la Tabla 6. Todos los profesores incorporan elementos descritos por ellos como novedosos, aquellos elementos más significativos y atingentes a las realidades escolares de cada uno.

\section{Evaluación Realizada por Pasantes al Término del Proceso}

Se observa en la Tabla 7 que los pasantes evaluaron con alta satisfacción los diferentes aspectos de la Pasantía, y en promedio, asignan 3.6 puntos de satisfacción general (escala Likert de 1 a 4, donde 4 representa mayor grado de satisfacción).

El 100\% señaló que recomendaría la Pasantía PENTA UC a otros profesores. El 90\% la considera mejor que otros perfeccionamientos, porque permite realizar un trabajo personal que surge de su motivación; se obliga a transferir los aprendizajes a través del proyecto y del compromiso de aplicación; hay mayor exigencia y se aprovecha el tiempo; y hay un constante seguimiento y retroalimentación del trabajo realizado. Algunos señalan que les permitió ver metodologías aplicadas y aprender así actividades concretas. Un pasante considera que es similar a otras experiencias de perfeccionamiento.

Tabla 5

Evaluación proyecto de transferencia

\begin{tabular}{ccccc}
\hline & $\begin{array}{c}\text { Auto evaluación } \\
\text { pasante }\end{array}$ & $\begin{array}{c}\text { Asesor } \\
\text { Disciplinario }\end{array}$ & $\begin{array}{c}\text { Asesor } \\
\text { Metodológico }\end{array}$ & Promedio \\
\hline Promedio & $94.4 \%$ & $83.3 \%$ & $77.8 \%$ & $83.9 \%$ \\
DS & $8.9 \%$ & $15 \%$ & $12.8 \%$ & $8.9 \%$ \\
\hline
\end{tabular}


Tabla 6

Elementos aprendidos por pasantes e incorporados a sus proyectos de transferencia

\begin{tabular}{|c|c|}
\hline Elementos incorporados & Actividades propuestas en los proyectos \\
\hline $\begin{array}{l}\text { Incorporación de recursos } \\
\text { pedagógicos }\end{array}$ & $\begin{array}{l}\text { Mapas conceptuales. } \\
\text { Análisis de canciones. } \\
\text { Pictogramas. } \\
\text { Lluvia de ideas. } \\
\text { Organizador gráfico. } \\
\text { Imaginerías. } \\
\text { Historias de personajes relevantes para el tema tratado. } \\
\text { Actividades de motivación al inicio de las sesiones. } \\
\text { Ejercicios de relajación. } \\
\text { Recursos tecnológicos: } \\
\text { - Uso de PWP. } \\
\text { - Búsqueda información en Internet. } \\
\text { - Videos. } \\
\text { - Softwares (RASMOL, Geómetra). } \\
\text { - Uso sala ENLANCES. }\end{array}$ \\
\hline Trabajo grupal de los alumnos & $\begin{array}{l}\text { Realización de investigaciones grupales. } \\
\text { Entrevistas grupales. } \\
\text { Diseño y desarrollo de proyectos en grupo. }\end{array}$ \\
\hline Actividades prácticas & $\begin{array}{l}\text { Experimentos. } \\
\text { Investigaciones. }\end{array}$ \\
\hline $\begin{array}{l}\text { Uso de otros espacios de } \\
\text { aprendizaje }\end{array}$ & $\begin{array}{l}\text { Incorporación de salidas a terreno. } \\
\text { Uso de laboratorio. }\end{array}$ \\
\hline $\begin{array}{l}\text { Espacios de expresión para } \\
\text { los alumnos }\end{array}$ & $\begin{array}{l}\text { Exposiciones. } \\
\text { Debates sobre temas de actualidad, temas de interés de los alumnos. }\end{array}$ \\
\hline Discusión de temas de actualidad. & $\begin{array}{l}\text { Relatar sueños, experiencias. } \\
\text { Exponer trabajos y discusión de ellos. }\end{array}$ \\
\hline $\begin{array}{l}\text { Trabajo estructurado previamente } \\
\text { por el profesor }\end{array}$ & $\begin{array}{l}\text { Guías de trabajo (presentadas en entrega del proyecto). } \\
\text { Cuadernillo de trabajo (presentado en entrega del proyecto). } \\
\text { Clases preparadas en transparencias. } \\
\text { Presentación en power point por parte del profesor. }\end{array}$ \\
\hline $\begin{array}{l}\text { Incorporación de estrategias } \\
\text { de evaluación }\end{array}$ & $\begin{array}{l}\text { Evaluación de los alumnos hacia los profesores. } \\
\text { Autoevaluación. } \\
\text { Evaluación entre pares. } \\
\text { Elaboración de pautas de evaluación a utilizar. } \\
\text { Corrección de trabajos o ejercicios, como actividad grupal en la clase. } \\
\text { Desarrollo de pautas de cotejo y rúbricas de evaluación. }\end{array}$ \\
\hline
\end{tabular}

Tabla 7

Promedio de satisfacción de pasantes

\begin{tabular}{lccccccccc}
\hline Aspecto & $\begin{array}{c}\text { Proceso } \\
\text { selección }\end{array}$ & $\begin{array}{c}2 \\
\text { pasantes } \\
\text { por curso }\end{array}$ & Lecturas & $\begin{array}{c}\text { Asesoría } \\
\text { Disciplinaria }\end{array}$ & $\begin{array}{c}\text { Asesoría } \\
\text { metodológica }\end{array}$ & $\begin{array}{c}\text { Clases con } \\
\text { alumnos }\end{array}$ & $\begin{array}{c}\text { Calidad } \\
\text { clases }\end{array}$ & $\begin{array}{c}\text { Calidad } \\
\text { docente }\end{array}$ & $\begin{array}{c}\text { Satisfacción } \\
\text { general }\end{array}$ \\
\hline $\begin{array}{l}\text { Nivel } \\
\text { satisfacción }\end{array}$ & 3.7 & 3.7 & 3.4 & 3.6 & 3.9 & 3.5 & 3.7 & 3.9 & 3.6 \\
\hline
\end{tabular}


Lo más destacado son las asesorías, el trabajo personalizado y la preocupación por su aprendizaje. Comentan que fue una oportunidad para aprender a hacer proyectos, diseñar clases, y concretar ideas. Como aspectos a mejorar se plantea que se debiera dar más tiempo para hacer el proyecto y tener más asesorías grupales para reflexionar con otros pasantes y conocer sus proyectos.

\section{Relación Entre Características de los Pasantes y sus Resultados en la Pasantía PENTA UC}

Se observó que no existen diferencias importantes por sexo ni dependencia en ninguno de los resultados obtenidos, es decir, ni en el nivel de aprendizaje de contenidos, de elementos metodológicos, en la calidad de los proyectos de transferencia ni en el nivel de satisfacción.

Sólo es posible apreciar leves diferencias por área disciplinaria del curso PENTA UC del cual participaron los pasantes, en nivel de aprendizaje de contenidos, siendo el porcentaje de logro mayor en física y matemática que en humanidades y ciencias sociales (30\% versus $10 \%$ aproximadamente). Con respecto al aprendizaje metodológico, los profesores de cursos de ciencias y ciencias biológicas señalan un número levemente superior de elementos novedosos (10 versus 7 u 8), lo que podría tener que ver con la posibilidad de acceder a uso de laboratorios y de tecnología, dando a conocer un aprendizaje científico que es más alejado de la realidad escolar. No se aprecian diferencias por área, en cuanto al nivel de satisfacción de los pasantes.

En cuanto a la calidad del proyecto, si bien tampoco existen diferencias importantes, se observa que los profesores que tomaron cursos de ciencias biológicas o ciencias, obtienen promedios levemente superiores que los de humanidades y ciencias sociales ( $89 \%$ versus $76 \%$ aproximadamente). Llama la atención que los pasantes de física y matemática obtienen el promedio más alto en el proyecto, y el más bajo en la prueba de conocimientos. Esto realza la importancia de realizar evaluaciones de los diferentes objetivos de aprendizaje de la Pasantía, dando importancia tanto a los aprendizajes metodológicos, como a los de conocimiento de contenidos.

\section{Discusión}

La Pasantía PENTA UC alcanza en gran medida los objetivos planteados, siendo una estrategia innovadora que aporta al desarrollo profesional docente. La evaluación muestra aprendizajes en los pro- fesores que se asocian a prácticas docentes efectivas: aumentan su nivel de conocimiento disciplinario, aprenden herramientas metodológicas, reflexionan sobre la propia práctica y el aprendizaje, y aprenden a elaborar un proyecto donde se plasman estos aprendizajes en una propuesta original y surgida de su motivación, adecuada a su realidad escolar.

Esta experiencia cuenta con elementos positivos que mostraron ser exitosos y que pueden aportar al planteamiento de otros perfeccionamientos. Aún cuando es acotada, muestra que es posible obtener resultados positivos, y que existen oportunidades para aprovechar experiencias pedagógicas de excelencia y aportar al desarrollo profesional docente. Los elementos principales a la base de los positivos resultados de la pasantía, se muestran en la Tabla 8.

Se consideran elementos teóricos descritos como efectivos, con el objetivo de contribuir al desarrollo profesional de los pasantes desde un espacio privilegiado, la educación de talentos académicos, donde existe un encuentro de excelencia, entre profesores expertos y motivados, con alumnos hábiles y motivados.

En general, los pasantes muestran aumentos en su nivel de conocimiento. Ahora bien, señalan que aprendieron contenidos, sin considerar los resultados de la evaluación final que muestran que éste es muy heterogéneo. Pareciera que utilizan otros criterios para hacer un juicio sobre sus aprendizajes, por ejemplo, el esfuerzo que implicó. Esto es importante de considerar en los perfeccionamientos pues los profesores deben saber qué mirar y qué criterios utilizar para evaluar.

Es destacable que el pasante participa de un programa de enseñanza completo, es decir, desde que se inicia el trabajo con los alumnos, hasta que se termina y evalúan los resultados, por tanto, se enriquece el aprendizaje y el análisis que pueden hacer de la experiencia.

La Pasantía PENTA UC desafía la visión de los pasantes quienes conciben la metodología como variedad de actividades. Si bien encontraron actividades novedosas, el aprendizaje más importante fue descubrir un modo de concebir el proceso de enseñanza - aprendizaje que es novedoso para ellos, lo que les permite crear y utilizar estrategias y actividades pedagógicas. Han reconocido la importancia de que el profesor maneje los contenidos en profundidad, siendo su rol estimular y acompañar el aprendizaje. Aprendieron a plantearse objetivos de aprendizaje claros, a confiar en los alumnos y darles libertad. 
Tabla 8

Principales componentes de la Pasantía PENTA UC

\begin{tabular}{ll}
\hline \multicolumn{1}{c}{ Aspecto positivo } & \multicolumn{1}{c}{ Descripción } \\
\hline Contexto de excelencia & Contexto exigente, donde se busca la excelencia por parte de académicos \\
& y de alumnos. Favorece la motivación de los pasantes, y plantea altas \\
& expectativas sobre ellos mismos y las posibilidades de mejoramiento en \\
& su quehacer docente. \\
\hline $\begin{array}{l}\text { Largo tiempo de aprendizaje y } \\
\text { en paralelo con su práctica }\end{array}$ & $\begin{array}{l}\text { El aprendizaje es cambio, y como tal, requiere de tiempo. Se cuenta con } \\
\text { tiempo para aprender, poner a prueba, preguntar, reintentar. Además, al }\end{array}$ \\
& ser un trabajo en paralelo con la práctica docente se favorece la transfe- \\
& rencia de lo aprendido. \\
\hline
\end{tabular}

Profesores comparten rol de alumno Pasantes toman la posición de los estudiantes escolares. Comparten sus con estudiantes escolares experiencias, vivencian lo que ellos hacen durante una clase y descubren o reconocen las respuestas que se generan en los estudiantes a partir de las diferentes acciones de los docentes, aspecto crucial a la hora de enseñar.

\begin{tabular}{ll}
\hline Aprendizaje por observación & Se ofrece la oportunidad de observar a un experto haciendo clases a \\
& escolares. Pasantes reconocen la experticia, y reconocen que se ven en- \\
& frentados a una situación similar a la que ellos enfrentan a diario, favore- \\
& ciendo así la autoridad de la experiencia y la riqueza del aprendizaje a \\
& partir de ella.
\end{tabular}
Aprendizaje por la acción $\quad$ Los pasantes participan de las actividades y la experiencia de aprendizaje, contrastando el nivel de adquisición de conocimientos. Surgen reflexiones, aprendizajes y cuestionamientos que se pueden ir resolviendo.

Asesorías y seguimiento Trabajo de asesoramiento individualizado y específico, que favorece un mayor conocimiento de contenidos, así como aprendizajes metodológicos y de elaboración de un proyecto. Estos espacios permiten resolver dudas, recibir apoyo y retroalimentación permanente. Se suman asesorías grupales que favorecen el intercambio de experiencias.

Diseño de un proyecto Herramienta profesional. Es un producto del aprendizaje muy valorado por los pasantes, pues se hace durante todo el proceso a partir de exigencias rigurosas, considerando la propia motivación y contexto escolar.

Flexibilidad dentro de los objetivos Si bien se plantean objetivos de aprendizaje para todos los pasantes, el para todos. proceso para llegar a ellos, y los productos, cuentan con flexibilidad para responder a los intereses y necesidades particulares, siendo una experiencia con sentido y posible para profesores de diferentes áreas y contextos.

Otra fortaleza es que los pasantes pueden observar a los docentes universitarios expertos en contenido que se ven inmersos en una situación similar a la que ellos enfrentan a diario, hacer clases a alumnos escolares, siendo entonces modelos válidos. La observación de situaciones de enseñanza-aprendizaje óptimas, permite a los profesores aprender elementos exitosos para el aprendizaje, lo que fortalece la fuerza del cambio (Bandura, 1983). Aprender a observar a otro con dichos marcos de referencia puede constituirse en una herramienta profesional de autoevaluación. Además, tiene la riqueza de que los profesores asistan a la universidad y vean un mundo diferente al contexto escolar.

Los profesores valoran aprender a diseñar un proyecto. Contar con una muestra concreta de su aprendizaje y un producto para transferir ese aprendizaje, es recomendable para la formación en servicio ya que permite sentirse productivo, capaz de crear y lograr metas, y volver a su establecimiento con muestras de su cambio. Esto, sumado a que las características de este proyecto permiten responder a 
los intereses y contexto particular de cada pasante, es una gran fortaleza de la Pasantía PENTA UC.

La elaboración del proyecto se traduce en una herramienta profesional no sólo por contar con clases planificadas, sino por el ejercicio de pensarlas, hacer propuestas y dirigir las acciones hacia una meta clara haciéndose responsable de los logros, lo que influirá en su manera de planificar sus clases futuras. Hacerse responsable de los resultados es una importante característica del desarrollo profesional y de los profesores efectivos (Arancibia, 1994; Núñez, 2004).

Los profesores reconocen que lo aprendido se puede incorporar a su realidad. Diseñar de manera concreta e intencionada un proyecto donde se conjugan las exigencias y herramientas proveídas por el perfeccionamiento con un compromiso y esfuerzo personal, es una fortaleza. Según Ávalos (2004), ofrecer propuestas metodológicas para enfrentar problemas no transforma las prácticas, se requiere que el profesor colabore en un diseño participativo.

El logro de buenos resultados en los pasantes, más allá de sus características de entrada, muestra que la Pasantía responde a la diversidad de necesidades particulares, dentro de un marco general donde se acompaña un proceso de aprendizaje. Los profesores pudieron reflexionar con respecto a lo que a ellos les hace sentido, observar a un profesor experto haciendo clases a escolares, asesorarse con él y con expertos en educación, acceder a bibliografía acorde a lo que ellos van realizando, y elaborar un proyecto que mezcla las exigencias con lo que ellos consideran importante e interesante. Así, la Pasantía PENTA UC permite flexibilidad para lograr los objetivos establecidos.

La literatura recomienda contar con espacios de reflexión pedagógica, y para ello, los profesores requieren de tiempo para observar, cuestionar, comprender e incorporar conocimientos (Barocio, 1993; Levine, 2002; Rodgers, 2002). La duración de un semestre en paralelo con la práctica profesional, permite decantar lo observado y experimentado, pensar sobre esa experiencia, re-mirar su propia práctica a la luz de estos elementos y discutirlos con expertos así como con sus pares.

Otra característica innovadora es que los pasantes toman la posición de alumnos siendo sus pares en el proceso de aprendizaje. Aprenden a mirar desde sus ojos, aumentando la comprensión de lo que necesitan, los motiva y son capaces de hacer. Los profesores reconocen que la buena enseñanza es una respuesta al aprendizaje en vez de la causa, y se vuelven curiosos y concientes del aprendizaje (Rodgers, 2002). Esta mirada ayuda a que pongan énfasis en lo académico, aprovechando las características y potenciales de los alumnos.

Por otra parte, los pasantes contaron con asesorías específicas para tratar diferentes temas, tanto de contenidos, como para la elaboración del proyecto. Este seguimiento y apoyo permanente es fundamental para obtener buenos resultados, pues fue una instancia que ofrece retroalimentación permanente desde expertos, así como entre pares, lo que fue motivante y desafiante para los pasantes.

Ahora bien, existen aspectos a considerar para replicar una experiencia como ésta. Se requiere de un equipo académico y metodológico de gran calidad, lo que encarece la implementación. Esta experiencia se realizó en el contexto de educación de talentos, en la que se cuenta con académicos expertos y clases de alto nivel, con alumnos altamente capaces y motivados. Esta riqueza se ejemplifica en planteamientos de los mismos alumnos del programa, quienes luego de participar de cursos con pasantes, señalan que el PENTA UC es una oportunidad para que los pasantes tengan más conocimientos, sean más completos, mejoren su desempeño y sus clases. Por ello, si bien algunas de sus características son replicables, conlleva el desafío de reconocer aquellos recursos pedagógicos innovadores y de excelencia existentes que permitan experiencias como éstas.

Cabe destacar que se trabaja con profesores motivados que eligieron participar, lo que favorece el logro de resultados positivos. Este elemento motivacional es de suma importancia, y hace difícil obtener resultados similares en grupos amplios de profesores que no deciden voluntariamente participar.

Dados los objetivos de esta investigación, se utilizó principalmente metodología cualitativa, junto con análisis cuantitativos descriptivos, por ello, al analizar los resultados, es importante atender a que no es posible hacer generalizaciones. Será un aporte continuar con evaluaciones, y desarrollar instrumentos que permitan profundizar y objetivar estos resultados, de modo de conocer con mayor precisión los factores que influyen en el aprendizaje de contenidos y de herramientas metodológicas, y cómo ello modifica creencias de los profesores sobre el proceso de aprendizaje. Además, es necesario continuar con evaluaciones en el tiempo pues es fundamental analizar el impacto en el aprendizaje de los estudiantes, para seguir con propuestas en esta línea. Lo que será realizado en un segundo estudio. 
En síntesis, es posible desarrollar ofertas de perfeccionamiento efectivas y de calidad, acompañadas de evaluación para mejorarlas, de manera de hacerse responsable de los resultados obtenidos. Es fundamental considerando el rol de los profesores en el logro de los objetivos educativos, especialmente en países como Chile, donde la educación es clave para mejorar la calidad de vida de sus ciudadanos y del país. Es un imperativo generar conocimiento y realizar un trabajo de perfeccionamiento que permita lograr aprendizajes en los docentes y transformar así sus prácticas, en beneficio del aprendizaje de sus estudiantes.

\section{Referencias}

Alliaud, A. \& Duschatzky, L. (Comps.). (1998). Maestros. Formación práctica y transformación escolar. Buenos Aires: Miño y Dávila Editores.

Arancibia, V. (1994). Formación y capacitación de los profesores: Impacto en el aprendizaje de los Estados Unidos. Proyecto Principal de Educación, 34, 51-76. Santiago: UNESCO/OREALC.

Arancibia, V. \& García, C. (2003). Diseño de un sistema de pasantias PENTA UC. Santiago: Proyecto FONDEF DO2I1039.

Arancibia, V., Herrera, P. \& Strasser, K. (1999). Psicología de la educación. México D.F.: Alfaomega.

Ávalos, B. (2004). La formación de profesores y su desarro1lo profesional. Prácticas innovadoras en busca de políticas. El caso de Chile. En C. Cox (Ed.), Políticas educacionales en el cambio de siglo. La reforma del sistema escolar de Chile (pp. 559-594). Santiago: Editorial Universitaria.

Bandura, A. (1983). Principios de modificación de conducta. Salamanca: Ediciones Sígueme.

Barocio, R. (1993). La formación docente para la innovación educativa. El caso de un currículum con orientación cognoscitiva. México D.F.: Trillas.

Bellei, C. (2001). ¿Ha tenido impacto la reforma educativa chilena? En C. Cox (Ed.), Politicas educacionales en el cambio de siglo. La reforma del sistema escolar de Chile (pp. 125-209). Santiago: Editorial Universitaria.

Bellei, C. \& Pérez, L. (Eds.). (2000). La renovación de la profesión docente. Ciclo de debates: Desafios de la politica educacional. Santiago: UNICEF.

Bransford, J., Brown, A. \& Cocking, R. (Eds.). (2000). How people learn: Brain, mind, experience and school [Versión electrónica]. Washington: National Academy Press.

Brunner, J. \& Elaqua, G. (2003). Informe capital humano en Chile. Santiago: Universidad Adolfo Ibáñez.

Fecha de recepción: Noviembre de 2005.

Fecha de aceptación: Julio de 2006.
Cashion, M. \& Sullegner, K. (2000). "Contact us next year": Tracing teachers' use of gifted practices. Roeper Review, 23(1), 18-21.

Ferrández, A., Gairín, J. \& Tejada, J. (1990). El proceso de aprendizaje en el adulto. Zaragosa: Felix Arilla, S.C.

Fullan, M. \& Stiegelbauer, S. (1997). El cambio educativo. México, D.F: Trillas.

Guskey, T. (2002). Professional development and teacher change. Teachers and teaching: Theory and practice, $8(3 /$ 4), 381-391.

Jones, M. (1997). Trained and untrained secondary school teachers in Barbados: Is there a difference in classroom performance? Educational Research, 39(2), 175-184.

Kerwin, M. \& Rhoads, J. (1996). Improving teaching through a peer support "teacher consultation process". Recuperado desde http://orders.edrs.com/members/sp.cfm

Levine, D. (2002). Thinking about doing. On learning from experience and the flight from thinking. Human Relations, 55(10), 1251-1268.

Lieberman, A. \& Miller, L. (1999). Teachers-transforming their world and their work. New York: Teachers College Press.

Livingston, C. \& Borko, H. (1989). Expert-novice differences in teaching: A cognitive analysis and implications for teacher education. Journal of Teacher Education, 36-41.

Marchesi, A. \& Martin, E. (1998). Calidad de la enseñanza en tiempos de cambio. Madrid: Alianza.

Núñez, I. (2000). Los docentes y la política de desarrollo profesional. Recuperado desde www.mineduc.cl

Núñez, I. (2004). El profesorado, su gremio y la reforma de los años noventa: Presiones de cambio y evolución de la cultura docente. En C. Cox (Ed.), Politicas educacionales en el cambio de siglo. La reforma del sistema escolar de Chile (pp. 455-517). Santiago: Editorial Universitaria.

OCDE. (2004). Revisión de políticas nacionales de educación: Chile. Organización para la cooperación y el desarrollo económico. Centro para la cooperación con países no miembros.

Project ExCELL. (1997). Professional development for language teachers. Austin, TX: Texas Education Agency.

Rodgers, R. (2002). Seeing student learning: Teacher change and the role of reflection. Harvard Educational Review, 72(2), 230-253.

Scheerens, J. (2000). Improving school effectiveness. Part II. Research: A review of the evidence from developed and developing countries. París: UNESCO-IIEP. Recuperado desde http://unesdoc.unesco.otg/images/0012/001224/ 12242e.pdf

Stiegler, J. \& Hiebert, J. (2002). La brecha de la enseñanza. Estudios Públicos, 86, 57-144.

Undurraga, C. (2004). ¿Cómo aprenden los adultos? Santiago: Ediciones Universidad Católica.

Van Der Sijde, P. (1989). The effect of a brief teacher training on student achievement. Teaching and Teacher Education, 5(4), 303-314. 